\title{
Familias: diversidades teóricas y políticas
}

Catalina Arteaga A. (Departamento de Sociología, Universidad de Chile). Lorena Armijo (Universidad Santo Tomás)

Este número monográfico de Punto Género titulado "Familias en transformación: diversidades y disputas" recoge seis artículos de investigación empírica los cuales, en su conjunto, problematizan la noción de familia y sus prácticas desde el debate que ha asignado la centralidad a las familias nucleares heteronormativas, hasta la periferia donde se instalan nuevos tipos de familias, alejadas del vínculo sanguíneo y el parentesco tradicionalmente entendido. Tanto el cuestionamiento o revisión conceptual, como el desplazamiento analítico sobre los temas y poblaciones analizados por las ciencias sociales, suponen una posibilidad de abrir nuevas rutas teóricas, así como subvertir la jerarquización desigualitaria del orden de género. Es así como este número dedicado al análisis de las familias actuales y sus relaciones, desde el enfoque de género, permite comprender realidades subalternas y las fluctuaciones en las vivencias más visibles.

Cabe precisar que el abanico de perspectivas, objetos y sujetos de estudio mencionado se debe en parte a la presencia de la interseccionalidad usada como herramienta de develamiento de las múltiples formas y expresiones que asume la desigualdad de género, a partir de sus ejes de caracterización y diferenciación. En quienes escriben este número, la interseccionalidad es una apuesta situada y contingente, que permite analizar la relación del género con la etnicidad, la sexualidad, la clase, la edad y el nivel de formación, como un engranaje complejo en los procesos de dominación. Pese a la multiplicidad de significados que trae la interseccionalidad, hay un interés común en estos artículos: todos analizan el eje producción/reproducción del orden de género, con lo cual se actualiza la pregunta acerca de las posibilidades realmente existentes de la transformación al interior de las familias y de la eficacia social de las acciones destinadas para su desestabilización.

Cada artículo representa una aproximación distinta al tema de este número, lo que permite descentrar los enfoques en el estudio de las familias, llevando la discusión a nuevos derroteros socio-simbólicos y legales, que dan cuenta de la polisemia actual derivada de los emergentes escenarios sociales. Antes de presentar los artículos de este número, esbozaremos algunas discusiones relevantes que se han dado en los estudios de la familia centrándonos en tres aspectos: los cambios en las familias en relación a procesos generales de la sociedad; el cuestionamiento a las nociones tradicionales de familias, en relación a dichos cambios; la diversidad de formas de hacer familia hoy, las cuales desestabilizarían los significados y prácticas de la familia heteronormativa, con sus propias búsquedas y resoluciones. 
Revista Punto Género N ${ }^{\circ} 9$. Junio de 2018

ISSN 0719-0417 / 03 - 12

\section{Debates en las transformaciones familiares actuales}

En los últimos años, los análisis sociodemográficos dan cuenta de las transformaciones en los hogares y las familias en el país y en la región (Arriagada, 2009; Arteaga, et.al., 2012). Dichos cambios se dan en el marco de procesos más amplios que tensionan las relaciones familiares. Las mudanzas en las configuraciones familiares; las contradicciones entre los discursos e imaginarios disponibles respecto de la familia (PNUD, 2002) y la parentalidad; las expectativas sociales a la vez que la persistencia de relaciones de género desiguales; las exigencias institucionales en torno a la familia en un contexto de crisis de los cuidados y el retraimiento del Estado en su provisión, producen tensiones entre los deseos de individualización y autonomía y las exigencias normativas y estatutarias (Araujo y Martuccelli, 2012) a las que sus miembros están constantemente expuestos.

Para algunos especialistas, la aceptación de nuevas formas de constitución y funcionamiento de las familias con posibilidades de autonomía y de reflexividad de todos sus miembros en la toma de decisiones, sería expresión de la propia modernidad (Valdés et. al, 2005), aunque ello se daría de manera desigual en diversos sectores socioeconómicos. Otros cuestionan la eficacia de la familia como un refugio ante los avatares de la modernidad, dado que el problema central radicaría en que éstas no cuentan con los recursos cognitivos, materiales y de sociabilidad para hacer frente a los nuevos problemas a los que están enfrentadas (Güell, 1999). Dentro de los nuevos desafíos, se encuentra la organización de la vida en forma más individual, imponiendo una ética de autorrealización, de éxito y de búsqueda de posicionamiento social (Arteaga, 2017), que tensiona los roles parentales en la crianza y socialización.

La evidencia señala que los cambios familiares se dan en las prácticas, así como en las obligaciones y experiencias parentales, lo cual desafía el discurso centrado en la familia heterosexual y el matrimonio biparental (Rivas, 2009). Dicho desafío se ha dado desde distintas vertientes. Desde los enfoques críticos de los estudios de la familia, se reconoce la existencia de diversas relaciones entre miembros con atributos de género, generación y relaciones de poder diversas, que pueden dar paso a vínculos de colaboración, pero también de conflicto (De Oliveira y Salles, 1989; Hartmann, 2000). Autoras como Fraser (1990) han señalado que la familia debe ser analizada como un sistema económico y no sólo como un espacio de relaciones afectivas y desinteresadas. La división sexual del trabajo deposita en las mujeres las tareas domésticas, siendo para ellas el hogar un lugar de trabajo, de intereses potencialmente conflictivos, que pueden desembocar en explotación y violencia.

Efectivamente, las principales críticas a la noción funcionalista de la familia, se dieron desde los estudios de género y la teoría feminista, cuestionando el carácter androcéntrico, heteronormativo, e inequitativo de dicha concepción, en la medida que naturalizaba la desigualdad de roles al interior de la familia, obviando el problema del poder (Hartmann, 2000), así como la historicidad y diversidad de formas y estructuras familiares, concentradas en los sectores medios y urbanos de la población.

Desde la economía feminista, se ahondó en su análisis en el marco del cuestionamiento a la idea de que el trabajo productivo-mercantil es la única fuente de valor y evidenciando la 
invisibilización del trabajo reproductivo (Carrasco, 2013) y el papel de este último en la economía capitalista (Federici, 2013). En dicho contexto, el trabajo no remunerado desarrollado al interior de las familias, llevado a cabo principalmente por mujeres, se ha sustentado en la ideología de género (Greenstein, 1996), sin reconocimiento material ni simbólico. Dicha carga, si bien es transversal a las culturas y ha permanecido intacta por siglos, hoy en día, a partir de la mayor inserción de las mujeres al mercado de trabajo (Arteaga, 2005; Armijo; 2016; Leiva, S. y Comelin, 2015); el aumento de la esperanza de vida; la disminución del tamaño de los hogares -entre otros-han llevado a algunos/as autores/as a hablar de la crisis de los cuidados.

Desde otro ángulo, consideramos que la cuestión de la familia en Chile se da en torno a una disputa (Araujo y Martuccelli, 2012), política e ideológica, la cual se evidencia a partir de la legitimación de nuevas modalidades de relación familiar, las que son débilmente visibilizadas en el marco regulatorio actual (Cienfuegos, 2017). Los estudios recientes acerca de nuevas configuraciones familiares, dan cuenta de sujetos emergentes, como las familias y formas de parentalidad homosexual (Robaldo, 2011); familias adoptivas (Salvo, 2016) y análisis de nuevas modalidades de parentalides (Fernández, 2015) -como se verá a lo largo de los artículos de este número-. Dicha emergencia se ha dado en el contexto de disputas políticas e ideológicas en el país, a propósito de las discusiones legislativas de los últimos años en torno a la ley de aborto en tres causales; ley de adopción; ley de identidad de género; ley antidiscriminación o ley Zamudio, ley de acuerdo de unión civil.

En dicho marco de discusión y emergencia de nuevas modalidades de hacer familias, la noción de parentesco se ha ido debilitando. La distancia entre sexualidad, procreación, alianza y filiación, visibiliza el aspecto relacional y deliberado del parentesco y lleva a los individuos a un ejercicio de auto-reflexión y autodefinición de una identidad familiar en la que ya la fuerza de la sangre no es lo fundamental (Gonzálvez y Salvo, 2015). Si bien el parentesco constituye y ha constituido un elemento importante para conceptualizar y comprender a la familia como un grupo que ha estado presente en todas las sociedades (Paez, 1984) y que se diferencia de otros grupos sociales, se avanza en la idea de que a partir del parentesco - como un hecho no sólo biológico y natural, sino también socioeconómico y sociocultural- se tejen relaciones y vínculos que incorporan procesos de interacción, comunicación, autoridad, organización doméstica y de cuidado que hacen parte de la dinámica de cada familia, donde se involucran sentimientos y emociones que permean la experiencia de vida familiar para cada sujeto (Zapata, 2018). Ello da paso a nuevas formas de entender a la familia, más allá del círculo de consanguinidad.

Las relaciones de género en las familias, también han sido cuestionadas, particularmente en el caso de las mujeres, cuestionando sus identidades y roles tradicionales, a partir de la búsqueda de la igualdad y el reconocimiento, problematizando su papel dentro de las familias y resituándolo en contextos cada vez más fluctuantes, en los que la autoridad paterna se ha debilitado (Castells, 1999). Asimismo, la relación entre maternidad y mujer se ha visto cuestionada, abriendo posibilidades a éstas de ser madres de diversas formas y ampliando el abanico de posibilidades y decisiones frente a cuándo y cómo serlo particularmente en sectores medios y urbanos (Salvo, 2016). Por su parte, los padres 
muestran un mayor involucramiento en actividades con los hijos, principalmente en el grupo más joven (Olavarría, 2004) y en sectores medios y altos.

En dicho marco, se han cuestionado los conceptos tradicionales de maternidad y paternidad, dando paso a una mirada que enfatiza en la construcción social y cultural de los mismos, en un contexto histórico particular (Fernández, 2015). Así, es posible advertir la existencia de transformaciones y significados específicos de la parentalidad en contextos culturales diversos. En nuestro país, algunos estudios han señalado el sentido de sacrificio de la maternidad (Murray, 2012) así como las diferencias de clase en el significado de los roles parentales (Valdés et. al., 2005). Desde esta perspectiva, la maternidad y la paternidad pueden ser pensadas como "un campo de prácticas y significaciones culturales en torno a la reproducción, al vínculo que se establece o no con la progenie y al cuidado de los hijos" (Fuller, 2000). En ambos casos, involucra un salto, una distancia respecto del mismo hecho biológico de engendramiento, pues supone una construcción que coloca -y arraigadeterminadas experiencias en un contexto social, político, económico y cultural específico (Libson, 2009).

Al analizar las familias desde la diversidad o desde el enfoque de género, se asume la existencia de dinámicas familiares que rompen los supuestos biologicistas del parentesco a partir de la disociación entre relaciones conyugales y relaciones filiales; las posibilidades de establecer lazos de filiación independientemente de tener una relación de pareja heterosexual u homosexual; la disociación por las técnicas de reproducción asistida con donantes del componente biológico, genético, social y legal lo que complejiza los roles parentales y la construcción de la maternidad/paternidad; la multiparentalidad y pluriparentalidad (Rivas y Rivas; 2008; De Singly, 2007) .

Esta redefinición del parentesco se refleja en el aumento de nuevas modalidades de configuraciones familiares como las llamadas recompuestas, resultado de uniones entre individuos ocurridas después de una o más separaciones matrimoniales o de experiencias de convivencia. Con ello se rearticulan las formas la relación con los hijos provenientes de relaciones anteriores, reformulando las concepciones estáticas de maternidad y paternidad apegadas a lo biológico y dando lugar a paternidades sociales y culturales. También en las familias homosexuales se han mostrado formas específicas de parentalidad (Robaldo, 2011). No obstante, se evidencia falta de reconocimiento de nuevas prácticas, lo cual incide en inequidades, desigualdades, estigmatización y exclusión de estas modalidades de familias y prácticas parentales (Cienfuegos, 2017; Herrera, 2017).

Los cambios en la noción de parentesco, abren asimismo posibilidades de cuestionar la construcción histórica de lo femenino vinculado a la maternidad (Knibiehler et. al., 2012). Esta proximidad entre lo femenino y lo maternal ha sustentado en parte la reclusión de las mujeres, así como la desigualdad respecto de los hombres. Sin embargo, algunas investigadoras concluyen que a lo largo de la historia no ha existido una concepción uniforme de lo maternal que tal como las identidades femeninas no responden a un modelo homogéneo, sino que se organizan a partir de exigencias y de prácticas sociales, por lo que la maternidad es susceptible de cambiar de una época a otra (Knibielher et al, 2012). Dichas posibilidades de transformación se encuentran vinculadas a una idea de la parentalidad 
desde la noción de construcción social, concibiendo ésta en términos variables histórica y socioculturalmente, ligada a condiciones sociales, así como a imaginarios (Parrini, 2000) y dimensiones normativas.

El debate precedente sitúa la producción académica del presente número. La selección de artículos incluye casos de familias de Chile, Argentina, México y Uruguay, provenientes de zonas urbanas y rurales, de capitales y regiones, abordándose interpretaciones institucionales y subjetivas. En una primera línea de estudios sobre la tradicional familia nuclear heterosexual encontramos el artículo titulado "Entre familiarismo explícito y opcional: Cambios en el régimen de cuidados de infancia en Chile" de William Vera y Carina De La Barra, que analiza el régimen de cuidados en primera infancia en Chile, a partir de la identificación de tres mecanismos de provisión: servicios extra-familiares, trabajo intrafamiliar, y tiempo para el cuidado. Los resultados muestran que el modelo chileno transita desde un familiarismo explícito a uno opcional, donde la equidad de género muestra un maternalismo atenuado y una segmentación de la equidad socioeconómica. Esta transición presenta las resistencias del modelo tradicional a avanzar hacia la corresponsabilidad o el social care, producto de un institucionalismo que refuerza el papel de las mujeres como madres. Quienes escriben afirman que el régimen chileno ofrece mayores oportunidades de desfamiliarización que en el pasado, es decir, mayor posibilidad de externalizar el cuidado de las familias, sin embargo, reconocen la existencia de oportunidades y recursos de conciliación segmentados según niveles socioeconómicos. Este artículo se suma al debate internacional (europeo y latinoamericano) acerca de la conformación del bienestar social y su articulación de manera novedosa con los intereses en torno a la equidad de género.

Un segundo artículo denominado "Transformaciones en las relaciones de género-familiares y la agencia de campesinas indígenas cafetaleras en cooperativa" de Ana Georgina López Zepeda, indaga en dichas transformaciones en los ambientes rurales del Ejido Zaragoza, en Chiapas. Es un intento por describir las dinámicas rurales de las familias indígenas destinadas a la generación de ingresos extra-domésticos y las posibilidades que tienen las madres indígenas con hijos de construir colectivamente (en una cooperativa) sus capacidades individuales. Este estudio muestra una versión de la crudeza del orden de género en las familias heterosexuales tradicionales, cuando las mujeres se mudan de su familia nuclear a la familia política y la asumen como una de las etapas más duras en sus vidas, por la sobrecarga de actividades y las expectativas que tenían cuando se unieron a sus parejas varones. Sin embargo, el orden de género puede subvertirse, según la autora, desde la agencia de estas mujeres, como un recoveco de posibilidad, en tanto pueda romper con la interiorización de las normas culturales asumidas y con la resistencia ante las condiciones de desigualdad. Este artículo es un aporte en tanto une los debates de la agencia social y la desigualdad de género en un plexo de toma de conciencia y reflexividad que al menos remueve el género.

En una segunda línea de estudios relativos a la constitución de las familias actuales podemos agrupar los cuatro artículos restantes. El primero se titula "Aquí nadie es más que 
nadie". Igualdad y familias en el debate sobre el "matrimonio igualitario" en Uruguay" de Diego Sempol, e indaga en las condiciones que facilitaron el reconocimiento del derecho a contraer matrimonio por las parejas del mismo sexo, así como en las nociones familia y matrimonio, y lo considerado como legítimo y deseable para el estado, la democracia y la sociedad. El autor reconoce que la aprobación de dicha ley fue un avance en la igualdad jurídica y el reconocimiento de muchas personas y, a la vez, un golpe importante a la heteronormatividad, ya que en la práctica permitió por fin a la subordinación jurídica y simbólica de las ciudadanías que habita la población LGTB. Sin embargo, el autor destaca que en la discusión conceptual no existiría una conquista tan clara como en la realidad, por el contrario, los dos polos opuestos de la reflexión académica no logran un consenso acerca de que la aprobación de este tipo de normas supone una superación de los roles de género y su jerarquización. Más allá de este disenso, existe la claridad de exponer el caso uruguayo como distinto al español y argentino donde se dictaron leyes similares, debido a que en estos países quedaron inconclusas (por ejemplo, la disposición de la herencia, el derecho a pensión en caso de separación o muerte o la adopción de infantes). Por lo tanto, el caso uruguayo sería exitoso a juicio del autor, ya que no sólo es un fuerte golpe al corazón del pensamiento conservador, sino que las acciones del movimiento social rompen la lógica partidocrática dominante en la política uruguaya. Se produce, así una desestabilización del monopolio de la movilización social (centrada en demandas de clase), la iniciativa legislativa y la construcción de nuevas agendas. Este artículo visibiliza de manera clarificadora el tránsito de las necesidades sociales hasta llegar a convertirse en demandas públicas que alcanzan la igualdad formal.

El segundo artículo de esta corriente se denomina "Familias homoparentales: ¿Reproducción o transgresión del género hegemónico?" de María Angélica Cruz y María Llantén Oyarce, busca comprender cómo las familias homoparentales reproducen y/o tensionan las normas hegemónicas del género y, si la participación en colectivos del movimiento homosexual favorece esas tensiones. Los resultados muestran una realidad dual, por un lado, las familias reproducen la familia nuclear tradicional con la presencia - al menos en los primeros años de vida de los hijos/as - de una imagen paterna masculina y una imagen materna femenina permanentes. $\mathrm{Y}$, por otra, entre quienes participan en el movimiento LGBTI, desarrollan prácticas que tensionan o desacatan las normas hegemónicas del género heteronormativo. Sin embargo, las autoras son enfáticas en afirmar que se trata de casos de maternidades lesbianas asistidas tecnológicamente, por lo que no puede generalizarse. También hay un intento por destacar el desacato al orden de género como una estrategia relevante del feminismo de la igualdad, pese a que las autoras se declaran desde el feminismo posestructuralista. Este artículo es una lectura del hacer performativo del género tras la búsqueda de la igualdad formal, con conquistas y retrocesos en disputas contingentes, que muestra el trasvasije conceptual de las búsquedas personales y colectivas en dicha materia.

El tercer artículo titulado "Relaciones de género emergentes y paternidad en el hacer familia dentro de contextos Homoparentales" de Marcelo Robaldo, quien afirma que las personas no heterosexuales pueden compartir una realidad comunitaria que los vincula solidariamente 
frente a la exclusión y, por ende, las lleva a "hacer" parentalidad de un modo reflexivo, no natural, lleno de negociaciones, acuerdos y compromisos que lo diferencian de la construcción heterosexual del parentesco. El autor destaca que se hace familia no sólo desde la consanguinidad y de la conyugalidad, sino que dentro de un contexto comunitario común. Serían las personas protagonistas del estudio quienes reflexionan su experiencia de parentalidad desde el reconocimiento y la legitimación de la corporalidad femenina, desde los afectos hacia el hijo engendrado, poniendo en primer lugar sus necesidades, y destacando la paternidad emocional del padre no biológico homosexual. Este artículo separa los debates del parentesco de los de homoparentalidad permitiendo un hacer novedoso, basado en el presente, como un ejercicio de libertad de sus agentes.

El cuarto y último artículo denominado "Familia "entre pares". Relaciones de solidaridad y vínculos de fraternidad entre travestis y transexuales de la Argentina contemporánea" de Mariana Álvarez Broz muestra la construcción del proceso de subjetivación trans que conduce a las personas de esta condición a abandonar el hogar familiar a una temprana edad y a establecer nuevos vínculos sociales y afectivos con otras travestis y transexuales, con quienes conformarán su nueva familia, estableciendo relaciones de solidaridad y vínculos fraternos. Aquí la familia es un vínculo fluctuante y en construcción que devuelve la cercanía emocional y legitimidad pérdida en la familia originaria, no es casual que se hable de devenir trans en tanto camino sinuoso por el que atraviesan estas personas a lo largo de sus recorridos biográficos. La nueva familia también la socializa en la construcción de su feminidad y en los códigos de la supervivencia callejera como así también las habilidades necesarias para moverse en el ambiente de la transprostitución. La autora destaca que en estos intercambios y redes se da un pacto entre feminidades, que puede comprenderse a la luz de lo que se conoce como sororidad. Este artículo estable la siguiente paradoja que de manera más amplia también subyace en el feminismo de la igualdad: si bien las redes sociales son redes de contención al devenir trans, al mismo tiempo, coadyuvan a la reproducción de prácticas de riesgo y exclusión social.

El conjunto de artículos no sólo ofrece información y análisis sobre el quehacer de las familias en distintos contextos, sino que devuelve el interés por afinar los conceptos y revisar los trasvasijes teóricos y disciplinares, cada vez más necesario para comprender nuestro campo de estudio. 


\section{Bibliografía}

Araujo, K., \& Martuccelli, D. (2012). Desafíos comunes. Santiago : LOM

Armijo, L. (2016). Ciudadanía y cuidado en España: El dilema trabajo/familia según las madres trabajadoras. Psicoperspectivas, 15(3), 87-100.

Arriagada, I. (2009). La diversidad y desigualdad de las familias latinoamericanas. Revista latinoamericana de estudios de familia, 1, 9-21.

Arteaga, C. (2017). Diversidad de experiencias posicionales en el contexto de las transformaciones en la estructura social chilena. Revista Sociológica (91). UAMAzcapotzalco, México.

Arteaga, C. (2005). Prácticas y representaciones en torno al trabajo de temporeras agrícolas en Chile", en: Marta Mier y Terán, Cecilia Rabell (coordinadoras), Jóvenes y niños, un enfoque sociodemográfico, Instituto de Investigaciones, Universidad Nacional Autónoma de México, FLACSO-México, Editorial Porrúa, 2005.Pags: 127-163.

Arteaga , C., Aranda , V., \& Sepúlveda , D. (2012). Diversificación de las estructuras familiares: caracterización de las convivencias en Chile. Revista de Sociología

Carrasco, C., (2013). El cuidado como eje vertebrador de una nueva economía. Cuadernos de Relaciones Laborales, 31(1), pp. 39-56.

Castells, Manuel (1999) La era de la información. Vol. II, El poder de la identidad. México.

Cienfuegos, J (2017), "Regulación normativa de formas familiares en Chile", Ponencia presentada en Seminario: Semánticas familiares: nombrando, identificando y practicando los vínculos, Junio 2017, Universidad Academia de Humanismo Cristiano

De Oliveira, O., \& Salles, V. (1989). Acerca del estudio de los grupos domésticos: un enfoque sociodemográfico. En O. De Oliveira, M. Pepin Lehalleur, \& V. Salles, Grupos domésticos y reproducción cotidiana. México: UNAM.

De Singly, F. (2007). Sociologie de la famille contemporaine, éditions A. Colin, 2004 (3ème édition entièrement refondue, A. Colin, 2007). Paris.

Federici, S., (2013). Revolución en punto cero. Trabajo doméstico, reproducción y luchas feministas. Primera ed. madrid: Traficantes de sueños. 
Revista Punto Género N9. Junio de 2018

ISSN 0719-0417 / 03 - 12

Fernández, P. (2015) Infancia, parentalidad y familia en el discurso del Estado de Chile sobre adopción. Una aproximación desde el género. Tesis para optar al grado de magíster en estudios de género y cultura en América Latina, mención Humanidades, Universidad de Chile.

Fraser, N. (1990). ¿Qué tiene de crítica la teoría crítica? Habermas y la cuestión del género. Teoría feminista y teoría crítica. Ensayos sobre la política de género en las sociedades del capitalismo tardío.

Fuller, N. (2000): "Significados y prácticas de paternidad entre varones urbanos del Perú". En: Paternidades en América Latina. Lima: Pontificia Universidad Católica del Perú.

Gonzálvez, H. \& Salvo, I. (2015) Monoparentalidades electivas en Chile: Emergencias, tensiones y perspectivas. Psicoperspectivas. 14 (2), 40-40

Greenstein, T. N. (1996). Husbands' participation in domestic labor: Interactive effects of wives' and husbands' gender ideologies. Journal of Marriage and Family, 58(3), 585 - 595.

Güell, Pedro (1999): Familia y modernización en Chile. En: www.desarrollohumano.cl.

Hartmann, H. (2000). La familia como lugar de lucha política, de género y de clase: el ejemplo del trabajo doméstico. En M. Navarro, \& C. Stimpson R., Cambios sociales, económicos y culturales. México: Fondo de Cultura Económico.

Herrera, F. (2017) La mamá soy yo "La Mamá soy yo: Maternidad y discapacidad en Chile", Workshop "Pensando la maternidad: experiencias y desafíos en Chile, España y Reino Unido" , UDP, abril, 2017.

Knibiehler, Y., Arena, F., Cid López, R. (2012) “La maternité à l'épreuve du genre. Métamorphoses et permanences de la maternité dans l'aire méditerranéenne" Ed. Presses de l'École des hautes études en santé publique.

Leiva, S. \& Comelin, A (2015) Conciliación entre la vida familiar y laboral: Evaluación del programa IGUALA en una empresa minera en la región de Tarapacá, Polis Revista Latinoamericana 42, 2015 Racismo en Chile: colonialismo, nacionalismo, capitalismo

Libson, M. (2009) La diversidad en las familias: Un estudio social sobre parentalidad gay y lesbiana. Buenos Aires

Murray, M. (2012) Childbirth in Santiago de Chile: Stratification, Intervention, and Child Centeredness, MEDICAL ANTHROPOLOGY QUARTERLY, Vol. 26, Issue 3, pp. 319-337, ISSN 0745-5194

Olavarría, J. (2004), ¿Dónde está el nuevo padre? Trabajo doméstico: de la retórica a la práctica. En: Familia y vida privada ¿Transformaciones, tensiones, resistencias o nuevos sentidos?, Valdés, X y Valdés, T (eds), FLACSO/CEDEM, Santiago. Págs. 215-250.

Parrini, R. (2000) Los poderes del padre: paternidad y subjetividad masculina, en J. Olavarría y R. Parrini, Masculinidad/es. Identidad, sexualidad y familia. Flacso, Univ Academia de H Cristiano, Santiago, 2000. 
Paez, G. (1984). Sociología de la familia. Bogotá: Universidad Santo Tomás.

Programa para el Desarrollo de Naciones Unidas. (2002). Nosotros los chilenos. Un desafió cultural. Santiago: PNUD. ONU.

Rivas, A. (2009). Pluriparentalidades y parentescos electivos. Revista de Antropología Social, 18, 7-19. Recuperado de: http://revistas.ucm.es/index.php/RASO/article/viewFile/RASO0909110007A/8801

Rivas, Ana María (2008), Las nuevas formas de vivir en familia: el caso de las familias reconstituidas. Cuadernos de Relaciones Laborales ISSN: 1131-8635 2008, 26, núm. 1 179202.

Robaldo, M. (2011) La homoparentalidad en la deconstrucción y reconstrucción de la familia. Aportes para la discusión; Revista Punto Género 171

Salvo, I. (2016) Construcción de la maternidad en adopciones monoparentales: mandatos, deseos y elecciones, Revista de Psicología Universidad de Chile, 25 (2), Santiago de Chile.

Valdés, X., Caro, P., Saavedra, R., Godoy, C., Rioja , T., \& Raymond, E. (2005). Entre la reinvención y la tradición selectiva: familia, conyugalidad, parentalidad y sujeto en Santiago de Chile". En X. Valdés, \& T. Valdés, Familia y vida privada. Santiago de Chile: Cedem, Flacso.

Zapata, Adriana (2018), Prácticas familiares a distancia en contextos de migración internacional materna y paterna. Tesis Programa de Doctorado en Ciencias Sociales. Santiago de Chile (inédita). 